\title{
Time-Variant Haptic Simulator Based on Fine and Fast Real-Time Discretization Using FDTD Method
}

\author{
Hirotaka Muto* Student Member, Yuki Yokokura* Member \\ Kiyoshi Ohishi* Fellow
}

(Manuscript received Jan. 15, 2019, revised June 20, 2019)

\begin{abstract}
This paper proposes fine and fast discretization, which is based on the Finite Difference Time Domain (FDTD) method without a matrix exponential function. The proposed method is more accurate than the conventional discretization based on the first-order Pade approximation. Moreover, compared with that of the conventional method, the computational cost of the proposed method is very small. Although the Pade approximation is impossible to realize broadband real-time control, the FDTD method achieves the broadband real-time control. The proposed method and the conventional Pade approximation are analyzed by the Taylor expansion in a symbolic expression. According to the theorical analysis, the error of the FDTD discretization method is smaller than that of the conventional method. Numerical simulations also compare the errors and computational cost associated with the proposed and conventional methods. The FDTD discretization method is applied in admittance control for rendering the time-variant haptic sensation. In the case of the conventional method, owing to the limitation due to computation cost, the parameter update is not completed within the sampling period. On the other hand, the FDTD method completes the discretization within the sampling period. The gain and phase characteristics of the proposed method are close to the ideal responses. The validity of the method is verified by an experiment involving linear motor systems.
\end{abstract}

Keywords: real-time discretization, time-variant system, FDTD method, multi-inertia resonance system, admittance control, hapticsimulator

\section{Introduction}

Many systems in the real world are modeled as timevariant systems, and are described by state differential equations. Robots, traveling cranes, tether satellites, engines, elevators, and airplanes have dynamic systems in which the state transition matrix, matrix $A$, is composed of time-variant parameters ${ }^{(1)(2)}$. The parameters of the robot change according to its own posture ${ }^{(3)}$. In Ref. (3), although the robot was modeled as a time-invariant system with fixed constant parameters, the time varying factors depending on the posture were robustly controlled by a sliding mode controller. On the other hand, a method of actively controlling against time variations has also been studied ${ }^{(4)(5)}$. In position control of the three orthogonal axes, the resonance frequency depends on the position of each axis. Vibration suppression has been realized by the variable gain according to the time-varying resonance frequency. However, it is necessary to calculate the timevarying gain in advance and store the gain table in the computer's memory. When large parameter fluctuation occurs, the preservation amount of the time-varying gain becomes remarkably large. Furthermore, the vibration suppression controller for elevators cranes and tether satellites, which have variable rope length, are expressed as time-variant parameters ${ }^{(6)(7)}$. As with the traveling crane, a method such as gain scheduling has been adopted according to the time-variant

\footnotetext{
* Nagaoka University of Technology

1603-1, Kamitomioka-machi, Nagaoka, Niigata 940-2188, Japan
}

parameter $^{(8)}$.

In these conventional studies, to implement the controller for a time-variant system, the calculation cost is reduced by using tables calculated in advance. However, the discretization of high-order systems in every sampling period has not been conducted owing to the calculation cost.

In a training simulator operated by a human, it is necessary to accurately construct the physical model and set the sampling time to be short. For example, a real-time flight simulator and driving simulator have been developed to reproduce the reaction force of control surfaces or steering wheels ${ }^{(9)(10)}$. In a rehabilitation robot that uses force feedback, because the virtual physical model in accordance with operation by the human is updated in real time, it is necessary to perform discretization in every sampling period ${ }^{(11)}$. In this case, it is necessary to adjust the size of the system so that the calculation time does not exceed the control sampling time.

The calculation cost increases in the implementation owing to the matrix exponential function. When the continuous state equation, including the physical parameters, is discretized, it is equivalently transformed into a matrix exponential function. The matrix exponential function is generally implemented on a computer by using the Pade approximation $^{(12)(13)}$. However, the relation between calculation precision and calculation cost is a tradeoff in the Pade approximation. Therefore, when the size of matrix A increases, the computation cost increases exponentially. For that reason, this paper applies the FDTD method ${ }^{(14)}$ to discretize a spring mass system with higher order, time varying parameters, and 
proposes the fast real-time discretization with a low calculation cost. Unlike the conventional method, the proposed method does not use a matrix exponential function and a table calculated in advance. In addition, one advantage of the proposed method is that the discrete-time state equation derived by the FDTD method is simply expressed by continuous physical parameters.

\section{Discretization of a Continuous System}

The continuous time state differential equation of the target system is shown as follows:

$$
\begin{aligned}
\dot{x}(t) & =A_{c} x_{c}(t)+B_{c} u(t) \\
y(t) & =C_{c} x_{c}(t) \cdots \cdots
\end{aligned}
$$

where $A, B$ and $C$ denote the system matrices. The subscript $c$ and $d$ represent continuous and discrete. $x, u$ and $y$ denote the state vector, input, and output signals, respectively.

2.1 Time-Invariant System The continuous time system is strictly discretized by the sampler and zero-order hold. The discrete time system is equivalently derived by the matrix exponential function as follows:

$$
\begin{aligned}
& x[i+1]=A_{d} x_{d}[i]+B_{d} u[i] \ldots \ldots \ldots \ldots \ldots \ldots \ldots \\
& y[i]=C_{d} x_{d}[i] \\
& A_{d}=e^{A_{c} T_{s}} \\
& B_{d}=\int_{0}^{T_{s}} e^{A_{c} \tau} d \tau B_{c} \\
& C_{d}=C_{c}
\end{aligned}
$$

where $T_{s}$ denote the sampling period. The state, input, and output are constant values in the sampling period, and are represented as $x_{c}(t)=x\left[i T_{s}\right], u(t)=u\left[i T_{s}\right]$, and $y(t)=y\left[i T_{s}\right]$, where $i$ denotes the discre time. In a time-invariant system, the discretization is performed only once before the control loop. Therefore, parameter changes of the system after discretization are not considered.

2.2 Time-Variant System The system matrices matrix $A_{c}(t)$ and matrix $B_{c}(t)$ of the continuous time differential equation including the time-variant parameter are expressed by the time functions as follows:

$$
\begin{aligned}
& x[i+1]=A_{d}[i] x_{d}[i]+B_{d}[i] u[i] \\
& y[i]=C_{d} x_{d}[i]+D_{d} u[i] \cdots \ldots \ldots \\
& A_{d}[i]=e^{A_{c}(t) T_{s}} \ldots \ldots \ldots \ldots \ldots \ldots \\
& B_{d}[i]=\int_{0}^{T_{s}} e^{A_{c}(t) \tau} d \tau B_{c} . \cdots \cdots
\end{aligned}
$$

When the internal parameters are changed depending on the time, it is necessary to discretize the system according to the parameter change. Thus, Eq. (8) to Eq. (11) should be calculated in every sampling period.

2.3 Example of an nth-Inertia Resonance System

As an example, the state equation of the nth-inertia resonance system with time-variant parameters is shown as follows:

$$
\left[\begin{array}{c}
\dot{f}_{n} \\
\ddot{x}_{n} \\
\vdots \\
\dot{f}_{1} \\
\ddot{x}_{1}
\end{array}\right]=\left[\begin{array}{ccccc}
0 & k_{n}(t) & & & \\
-\frac{1}{m_{n}(t)} & 0 & & 0 & \\
& & \ddots & & 0 \\
& 0 & & 0 & k_{1}(t) \\
& & & -\frac{1}{m_{1}(t)} & 0
\end{array}\right]\left[\begin{array}{c}
f_{n} \\
\dot{x}_{n} \\
\vdots \\
f_{1} \\
\dot{x}_{1}
\end{array}\right]
$$

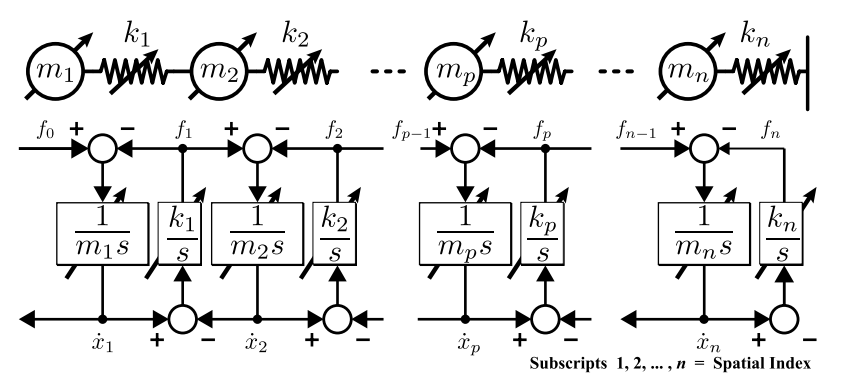

Fig. 1. nth-inertia environmental model with timevariant parameters

$$
\begin{aligned}
& +\left[\begin{array}{lllll}
0 & 0 & \cdots & 0 & \frac{1}{m_{1}(t)}
\end{array}\right]^{T} u(t) \cdots \cdots \cdots \cdots \\
& y=\left[\begin{array}{lllll}
0 & 0 & \cdots & 0 & 1
\end{array}\right]\left[\begin{array}{c}
f_{n} \\
\dot{x}_{n} \\
\vdots \\
f_{1} \\
\dot{x}_{1}
\end{array}\right] \ldots \ldots \ldots \ldots \ldots \ldots \ldots
\end{aligned}
$$

where $k$ and $m$ stand for time-variant stiffness and timevariant mass, respectively. The input, output, and each parameter are assumed as constant values within the sampling interval. Figure 1 shows the mechanical configuration and the block diagram of the nth-inertia resonance system. Ideal discretization based on analytic solutions is difficult to implement as the solutions are long and complicated. In the next section, Pade approximations are shown, and the problems are summarized.

\section{Conventional Discretization Method}

As a conventional method, the $l$ th order Pade approximation is shown.

3.1 Pade Approximation The Pade approximation of the matrix exponential function $e^{H}$ is expressed as follows:

$$
e^{H} \cong\left(I+\alpha_{1} H+\cdots+\alpha_{l} H^{l}\right)^{-1}\left(I-\alpha_{1} H+\cdots-\alpha_{l} H^{l}\right)
$$

where $\alpha$ and $l$ denote the coefficient of the Pade approximation and order $l$, which are given by

$$
\alpha_{l}=\alpha_{l-1} \frac{l-j+1}{j(2 l-j+1)}, \quad\left(\alpha_{0}=1, j=1,2,3, \cdots, l\right) .
$$

In this paper, the system is discretized by the first-order Pade approximation. The first-order Pade approximation is derived by $A_{c}$ as follows:

$$
A_{\text {Pade }}=\left(I-\frac{T_{s}}{2} A_{c}(t)\right)^{-1}\left(I+\frac{T_{s}}{2} A_{c}(t)\right), \quad\left(H=A_{c} T_{s}\right)
$$

Although the higher order approximation improves accuracy, matrix $A_{\text {pade }}$ becomes very long and complicated.

3.2 Problem of Conventional Method The firstorder Pade approximation is widely used for low-order matrix exponential functions. However, in higher order models, the computational cost increases exponentially. Furthermore, the accuracy also deteriorates. When using the Pade 
approximation, the implementation is limited owing to the trade-off relation between the discretization time and accuracy. As the order $l$ increases, the accuracy of the matrix exponential function increases. Therefore, it is possible to accurately discretize a control system designed in the continuous time domain. However, the absolute value of the computational cost is drastically increased. When performing a high-precision Pade approximation, it is necessary to set a long control period; therefore, it is not possible to implement a high-bandwidth control system.

\section{Proposed Real-time Discretization}

4.1 FDTD Method The FDTD method has been used for electromagnetic analysis of metamaterials and simulations of the sound field ${ }^{(15)(16)}$.

The algorithm of the FDTD method is applied to the spring-mass system shown in Fig. 1. The FDTD method is based on the wave equation. Therefore, it is effective for discretization of a multi-inertia spring mass system. The modeling in this paper is based on the following standard "wave equations":

$$
\begin{aligned}
& \frac{\partial \dot{x}(t, x)}{\partial t}=-\frac{1}{m_{n}(t)} \frac{\partial f(t, x)}{\partial x} \\
& \frac{\partial f(t, x)}{\partial t}=-k_{n}(t) \frac{\partial \dot{x}(t, x)}{\partial x} \cdots
\end{aligned}
$$

which are expressed as "partial derivatives" of the time and space(position) instead of simple Hooke's law $(f(t)=$ $\left.k_{n}(t) x(t)\right)$.

In this paper, each subscript and element index of the force are respectively defined as follows:

$$
\left.f_{\text {spatial index }} \text { [time index }\right] \text {. }
$$

As with the force, the subscript and element index of the velocity is also defined. Each differential equation is discretized by the algorithm of the FDTD method as follows:

$$
\begin{aligned}
\frac{\dot{x}_{n}[i+1]-\dot{x}_{n}[i]}{\Delta t} & =-\frac{1}{m_{n}^{\prime}[i]} \frac{f_{n}[i]-f_{n-1}[i]}{\Delta x} \cdots \\
\frac{f_{n}[i+1]-f_{n}[i]}{\Delta t} & =-k_{n}^{\prime}[i] \frac{\dot{x}_{n+1}[i]-\dot{x}_{n}[i]}{\Delta x} . \cdots
\end{aligned}
$$

The relationship between each element parameters and $\Delta x$ is as follows:

$$
\begin{aligned}
& m_{n}[i]=m_{n}^{\prime}[i] \Delta x \\
& k_{n}[i]=\frac{k_{n}^{\prime}[i]}{\Delta x} \cdots
\end{aligned}
$$

Eq. (20) and Eq. (21) are rewritten as follows:

$$
\begin{aligned}
\dot{x}_{n}[i+1]= & \dot{x}_{n}[i]+\frac{T_{s}}{m_{n}[i]}\left\{f_{n-1}[i]-f_{n}[i]\right\} \cdots \cdots \\
f_{n}[i+1]= & f_{n}[i] \\
& +T_{s} k_{n}[i]\left\{\dot{x}_{n}[i+1]-\dot{x}_{n+1}[i+1]\right\} \cdots
\end{aligned}
$$

where $f$ and $\dot{x}$ denote the force and velocity, respectively. $\Delta t$ is equivalent to $T_{s}$. The subscript $n$ stands for the numbering of the multi-inertia. The calculated value of the velocity is used for the force expression within the same control period. In the FDTD method, the velocity and force are alternately calculated.

The discrete equations of the FDTD method, discrete equations are implemented by applying the time-variant analog parameters $m_{n}[i]$, and $k_{n}[i]$ to Eq. (24) and Eq. (25). It is not necessary to consider the matrix exponential function as in the conventional method. Nevertheless, the calculation cost is small with a simple discretization.

4.2 State Equation of the FDTD Method Equations (24) and (25) are generally transformed into a state equation. Substitute Eq. (24) for $\dot{x}_{n}[i+1]$ of Eq. (25) as follows:

$$
\begin{aligned}
\dot{x}_{n}[i+1]= & \frac{T_{s}}{m_{n}[i]} f_{n-1}[i]+\dot{x}_{n}[i]-\frac{T_{s}}{m_{n}[i]} f_{n}[i] \cdots \\
f_{n}[i+1]= & \frac{k_{n}[i] T_{s}^{2}}{m_{n+1}[i]} f_{n+1}[i]-k_{n}[i] T_{s} x_{n+1}[i] \\
& +\left(1-\frac{k_{n}[i] T_{s}^{2}}{m_{n}[i]}-\frac{k_{n}[i] T_{s}^{2}}{m_{n+1}[i]}\right) f_{n}[i] \\
& +k_{n}[i] T_{s} x_{n}[i]+\frac{k_{n}[i] T_{s}^{2}}{m_{n}[i]} f_{n-1}[i] . \cdots
\end{aligned}
$$

As an example, the continuous expression Eq. (12) is discretized by the FDTD method as follows:

$$
\begin{aligned}
& x[i+1]=A_{F D T D}[i] x[i]+B_{F D T D} u[i] . \\
& y[i]=C x[i] \\
& A_{F D T D}[i]= \\
& {\left[a_{1,1}[i] \quad a_{1,2}[i\right.} \\
& a_{2,1}[i] \quad a_{2,2}[i \\
& 0 \\
& 0 \\
& a_{2 n-1,2 n-1}[i] \quad a_{2 n-1,2 n}[i] \\
& a_{2 n, 2 n-1}[i] \quad a_{2 n, 2 n}[i] \\
& a_{1,1}[i]=1-\frac{k_{n}[i] T_{s}^{2}}{m_{n}[i]} \ldots \ldots \ldots \ldots \ldots \ldots \ldots \ldots \\
& a_{1,2}[i]=k_{n}[i] T_{s} \\
& a_{2,1}[i]=-\frac{T_{s}}{m_{n}[i]} \\
& a_{2,2}[i]=1 \\
& a_{2 n-1,2 n-1}[i]=1-\frac{k_{1}[i] T_{s}^{2}}{m_{1}[i]}-\frac{k_{1}[i] T_{s}^{2}}{m_{2}[i]} . \\
& a_{2 n-1,2 n}[i]=k_{1}[i] T_{s} \\
& a_{2 n, 2 n-1}[i]=-\frac{T_{s}}{m_{1}[i]} \\
& a_{2 n, 2 n}[i]= \\
& B_{F D T D}[i]=\left[\begin{array}{lllll}
0 & 0 & \ldots & \frac{k_{1}[i] T_{s}^{2}}{m_{1}[i]} & \frac{T_{s}}{m_{1}[i]}
\end{array}\right]^{T} \\
& C=\left[\begin{array}{lllll}
0 & 0 & \cdots & 0 & 1
\end{array}\right] \\
& x[i]=\left[\begin{array}{lllll}
f_{n}[i] & \dot{x}_{n}[i] & \cdots & f_{1}[i] & \dot{x}_{1}[i]
\end{array}\right] .
\end{aligned}
$$

The FDTD method of Eq. (26) and Eq. (27) and the state equation are completely equivalent.

The FDTD discretization method is simply expressed by the continuous physical parameter. The FDTD method does not used the matrix exponential function, so calculation time is small due to direct discretization of the wave equation. The FDTD method completes the discretization within a sampling period. 


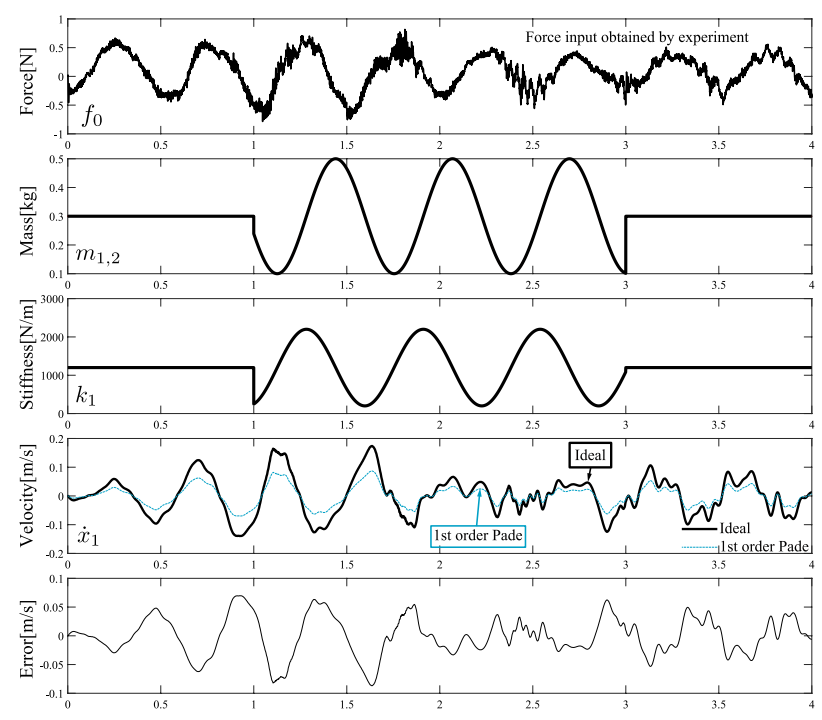

Fig. 2. Simulation of 1st Pade approximation(conv.): (i) Force input obtained by experiment, (ii) Time-variant parameter $m_{1}$ and $m_{2}$, (iii) Time-variant parameter $k_{1}$, (iv) Velocity responses $\dot{x}_{1}$, (v) Error between 1st Pade approximation and the ideal responses

\section{Numerical Validation}

The responses of the Pade approximation and proposed method are compared by simulations.

5.1 Simulations Setup The state equation of Eq. (12) is discretized at each sampling time. The force obtained from the experimental results is used as the input for the simulations. The parameters $m_{1}, m_{2}$, and $k_{1}$ are set to be time-variant from $1 \mathrm{~s}$ to $3 \mathrm{~s}$. The parameters of each time domain are as follows:

Time-invariant phase $\left(i T_{s} \leq 1 s\right)\left(3 s<i T_{s}\right)$;

$$
\left\{\begin{array}{l}
k_{1 . .6}\left[i T_{s}\right]=1200 \mathrm{~N} / \mathrm{m} \\
m_{1 . .6}\left[i T_{s}\right]=0.3 \mathrm{~kg}
\end{array}\right.
$$

Time-variant phase $\left(1 s<i T_{s} \leq 3 s\right)$;

$$
\left\{\begin{aligned}
k_{1}\left[i T_{s}\right] & =1000 \cos \left(0.001 i T_{s}\right)+1200 \mathrm{~N} / \mathrm{m} \\
m_{1 . .2}\left[i T_{s}\right] & =0.2 \sin \left(0.001 i T_{s}\right)+0.3 \mathrm{~kg} \\
k_{2 . .6}\left[i T_{s}\right] & =1200 \mathrm{~N} / \mathrm{m} \\
m_{3 . .6}\left[i T_{s}\right] & =0.3 \mathrm{~kg} .
\end{aligned}\right.
$$

5.2 Comparison of Simulation Results The velocity responses $\dot{x}_{1}$ of each discretization method are shown in Figs. 2 and 3. The ideal velocity responses are the thirteenthorder Pade approximation. The errors are the differences between the results of each discretization method and the ideal response. Figure 4 shows the errors between the discretization methods. The vertical axis is the square of the error, which should be a small value for the accuracy of the discretization to be high. Because the first-order Pade approximation has a large error, the accuracy of the discretization becomes low. On the other hand, because the FDTD method has a small error, the accuracy of discretization becomes high. The FDTD method does not degrade the accuracy of higher order systems.

5.3 Evaluation of Discretization Time To ensure real-time control, this paper uses the Linux software named

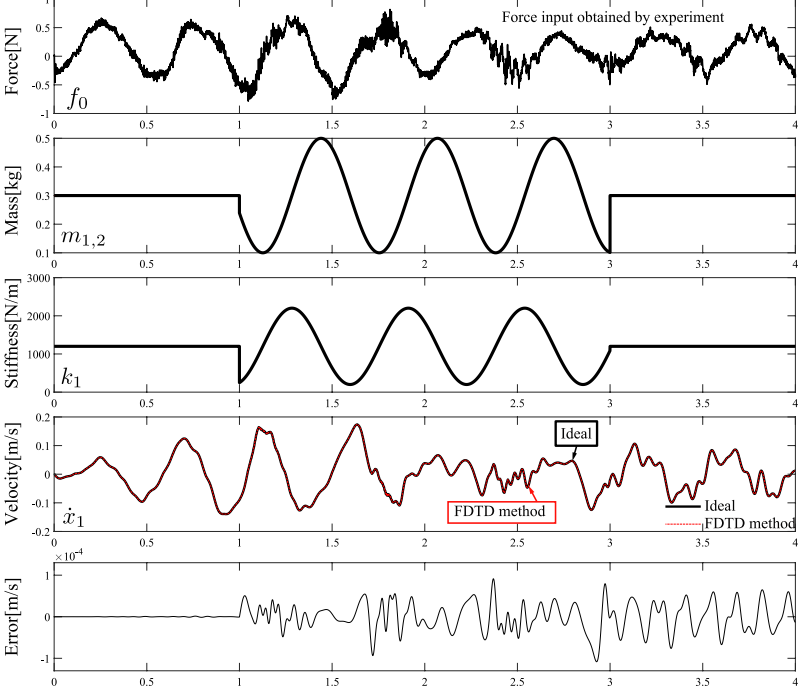

Fig. 3. Simulation of FDTD method(prop.): (i) Force input obtained by experiment, (ii) Time-variant parameter $m_{1}$ and $m_{2}$, (iii) Time-variant parameter $k_{1}$, (iv) Velocity responses $\dot{x}_{1},(\mathrm{v})$ Error between FDTD method and the ideal responses

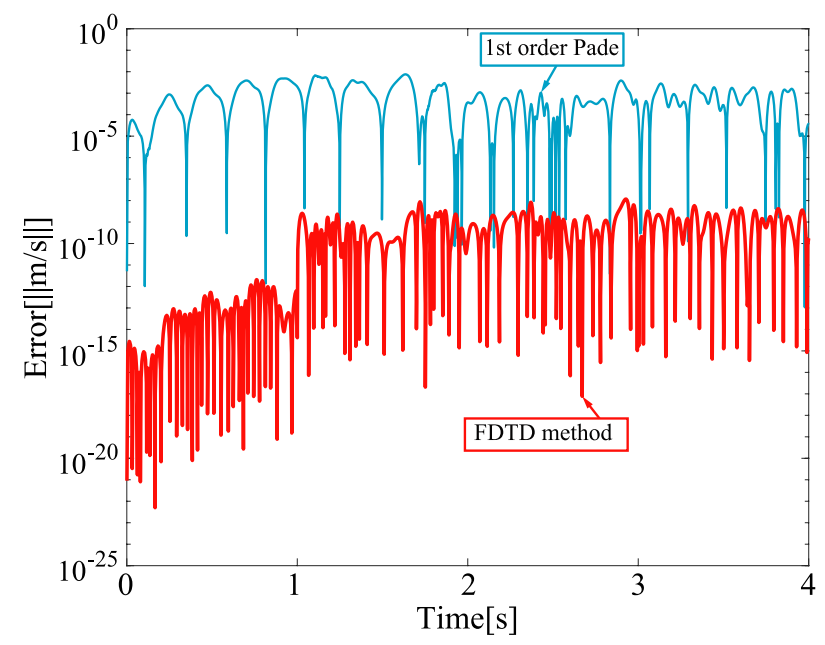

Fig. 4. Squaring error between 1st Pade approximation and FDTD method

as Advanced Robot Control System (ARCS). The specifications of the computer on which ARCS is implemented are Intel(R) Core(TM) i7-4770S at 3.10 GHz, memory 3.7 GB. In this paper, the control period is set to $100 \mu \mathrm{s}$. The calculation cost on ARCS is $6952 \mu \mathrm{s}$ in the conventional method and $78 \mu \mathrm{s}$ in the proposed method. Therefore, the calculation is completed within only $100 \mu \mathrm{s}$ in the proposed method.

This paper changes the number of the mess to and compare the discretization time. The discretization time of the 1to 10-inertia spring mass systems are shown in Fig. 5. Matrix $A_{c}$ of the n-th inertia spring-mass system is a square matrix with $2 n$ rows and $2 n$ columns. The Pade approximation increases the discretization time exponentially when the matrix $A_{c}$ increases. In the case of the six inertia spring mass system, matrix $A_{c}$ is an twelfth order state transition matrix. The discretization time of the six-inertia spring mass system is shown in the Table 1 . When the sampling time is $100 \mu \mathrm{s}$, calculation of the first-order Pade approximation is 


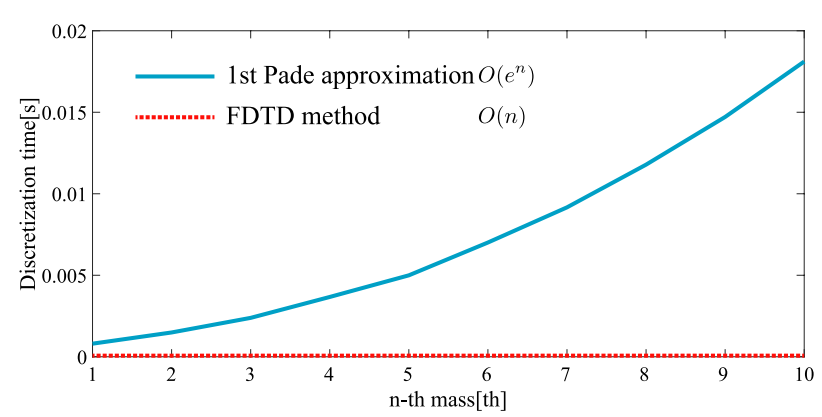

Fig. 5. Discretization time of 1 st pade approximation and FDTD method. Calculations are performed using ARCS that runs in a single thread

Table 1. Computation time for discretizing six inertia spring mass system by using the ARCS

\begin{tabular}{|c|c|c|}
\hline Method & 1st Pade appox. & FDTD method \\
\hline Discretization time [s] & $6952 \mu \mathrm{s}$ & $68 \mu \mathrm{s}$ \\
\hline
\end{tabular}

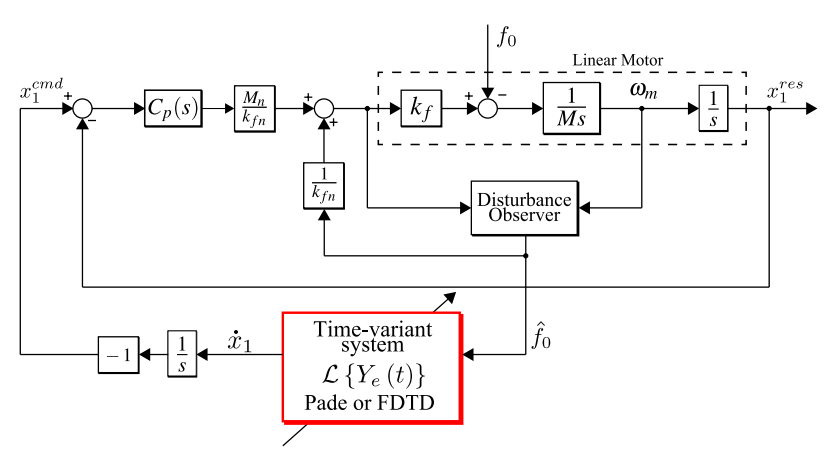

Fig. 6. Admittance control for haptic rendering

not completed within the control period. On the other hand, the proposed method completes the discretization within $100 \mu \mathrm{s}$.

\section{Application for Haptic Simulator}

This paper applies the FDTD real-time discretization method to the haptic simulator, which reproduces the haptic sensation of the target object in the virtual space. As the shape of the object changes, the internal mass and stiffness parameters also change. Therefore, the time-variant environment is discretized by the FDTD method to realize a highspeed simulator directly by using the analog parameters.

6.1 Admittance Control for Rendering The virtual mechanical impedance is reproduced by implementing admittance control on the liner motor ${ }^{(17)}$. The time-variant environmental admittance $\mathcal{L}\left\{Y_{e}(t)\right\}$ expressed by the discrete time state equation is virtually reproduced via the linear motor. The structure of admittance control for rendering the environment is shown in Fig. 6. $G_{A}(s)$ is the transfer function from the external force $f_{0}$ to the position response $x_{1}^{\text {res }}$ :

$$
\begin{aligned}
G_{A}(s)= & \frac{x_{1}^{r e s}(s)}{f_{0}(s)} \\
= & -\frac{\mathcal{L}\left\{Y_{e}(t)\right\}}{s} \frac{1}{s^{2}+C_{p}(s)}\left\{C_{p}(s) G_{o b s}(s)\right. \\
& +\frac{s}{\mathcal{L}\left\{Y_{e}(t)\right\} M_{n}}\left(1-G_{o b s}(s)\right\} . \cdots \ldots \ldots
\end{aligned}
$$

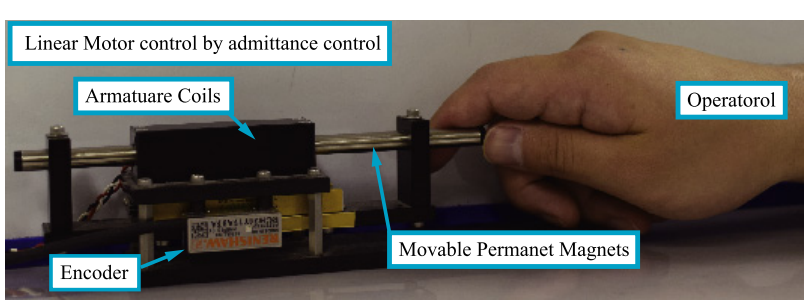

Fig. 7. Actual configuration for haptic simulator

Table 2. Setup parameter for conventional and proposed method

\begin{tabular}{|c|c|c|c|}
\hline Param. & Descriptions & Conv. & Prop. \\
\hline \hline$M_{n}$ & Mass of linear motor[kg] & 0.35 & 0.35 \\
$K_{f n}$ & Force constant[N/A] & 4.17 & 4.17 \\
$g_{p d}$ & Bandwidth of the pseudo differentiator[rad/s] & 300 & 3000 \\
$g_{\text {obs }}$ & Bandwidth of the DOB and RFOB[rad/s] & 150 & 1300 \\
$T_{s}$ & Sampling time [ $\mu \mathrm{s}]$ & 7000 & 100 \\
& Resolution of the linear encoder [nm] & 100 & 100 \\
\hline$g_{p}$ & PD controller poles [rad/s] & 30 & 200 \\
\hline$K_{p}$ & Proportional gain of the PD controller[1/ $\left.{ }^{2}\right]$ & 900 & 40000 \\
$K_{v}$ & Differential gain of the PD controller[1/s] & 60 & 400 \\
\hline$Y e(s)$ & Six inertial environments for rendering \\
\hline$m_{e}$ & Mass of environments [kg] & 0.3 & 0.3 \\
$k_{e}$ & Stiffness of environments [N/m] & 700 & 700 \\
\hline
\end{tabular}

$G_{o b s}(s)$ and $C_{p}(s)$ represent the disturbance observer (DOB) and position controllers, which are given by

$$
\begin{aligned}
& G_{o b s}(s)=\frac{\hat{f}_{0}(s)}{f_{0}(s)}=\frac{g_{o b s}}{s+g_{o b s}}, \cdots \ldots \ldots \\
& C_{p}(s)=K_{p}+K_{v} s=K_{p}+K_{v} \frac{g_{p d} s}{s+g_{p d}}
\end{aligned}
$$

where $g_{p d}$ denotes the Bandwidth of the differentiator. $K_{p}$ and $K_{v}$ denote the position proportional gain and the position integral gain, respectively.

6.2 Experimental Setup This paper implements the time-variant environment discretized by the FDTD method to admittance control. The force estimated by the DOB is input into the environment. The environmental velocity $\dot{x}_{1}$ of the motor with admittance control is then measured by the optical encoder. An overview of the experimental setup is shown in Fig. 7, and the experimental parameters are shown in Table 2. The time-variant parameters are the same as those of the simulations. The sampling period of the Pade approximation and the FDTD method are $7000 \mu$ s and $100 \mu$ s, respectively.

6.3 Experimental Results Figures 8 and 9 show the results of actual experiments with the Pade approximation and the FDTD method. In the conventional method, it is impossible to realize broadband admittance control with a sampling period of $100 \mu$ s The estimation of the force applied by the operator is shown at the top of the figure. The operator feels the reaction force from the time-variant environment. The first-order Pade approximation has a large error, the accuracy of the discretization becomes low. On the other hand, because the FDTD method has a small error, the accuracy of discretization becomes high. In the proposed method, the time-variant system is discretized in real-time, and admittance control is realized.

6.4 Evaluation of High-definition Frequency Responses The frequency response of each method is measured by the Frequency Response Analysis (FRA) ${ }^{(18)}$. The sampling time of each method depends on the calculation 


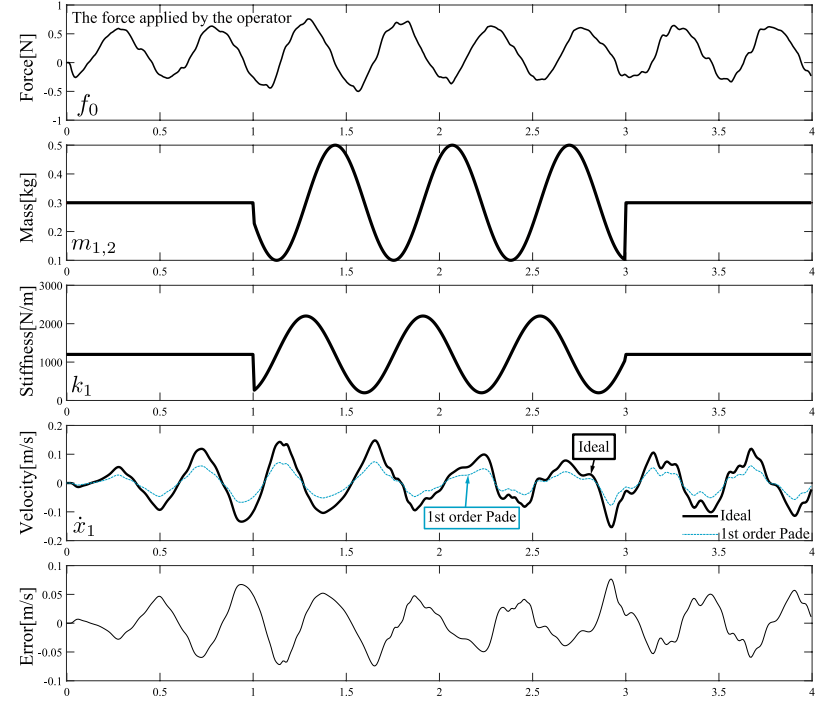

Fig. 8. Experimental results of the Pade approximation $\left(T_{s}=7000 \mu \mathrm{s}\right)$ : (i) Force estimated by disturbance observer, (ii) Time-variant parameter $m_{1}$ and $m_{2}$, (iii) Timevariant parameter $k_{1}$, (iv) Velocity responses $\dot{x}_{1}$, (v) Error between the FDTD method and the ideal response

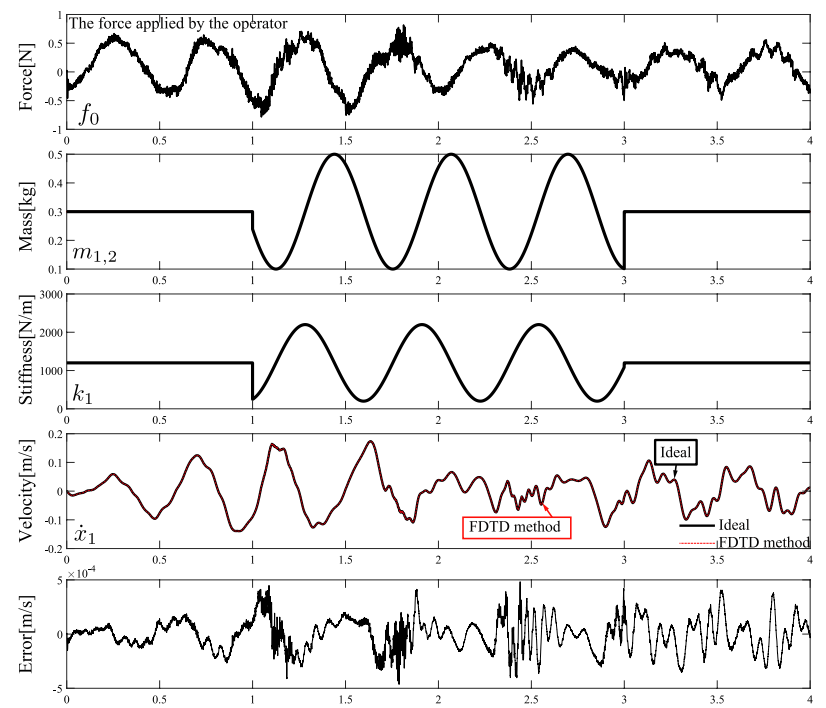

Fig. 9. Experimental results of FDTD method $\left(T_{s}=\right.$ $100 \mu \mathrm{s}$ ): (i) Force estimated by disturbance observer, (ii) Time-variant parameter $m_{1}$ and $m_{2}$, (iii) Time-variant parameter $k_{1}$, (iv) Velocity responses $\dot{x}_{1}$, (v) Error between the FDTD method and the ideal response

cost. Therefore, the bandwidth of each controller is limited by the sampling time. Note that the environments are set to be time invariant for the linear frequency response measurement. The set up parameters are shown in Table 2. The counter motor for the external force input is connected with the motor with admittance control via a coupling in the Fig. 10. The frequency responses from the external force to the position response in Eq. (39) are shown in the Fig. 11. In the conventional method, the anti-resonant frequency appears at different points. In addition, the phase is also shifted to a low frequency. The gain and phase of the proposed method are closed to the ideal responses. According to the conventional method, owing to the limitation of calculation cost, the parameter update does not end within a $100 \mu$ s sampling

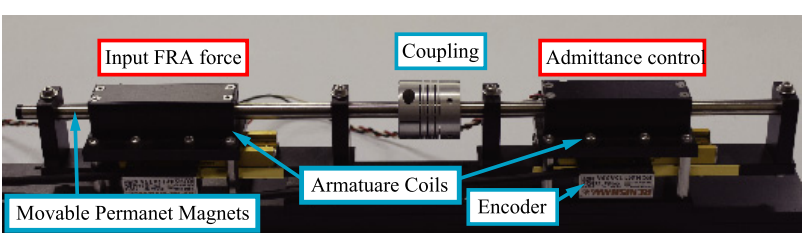

Fig. 10. Actual configuration for FRA
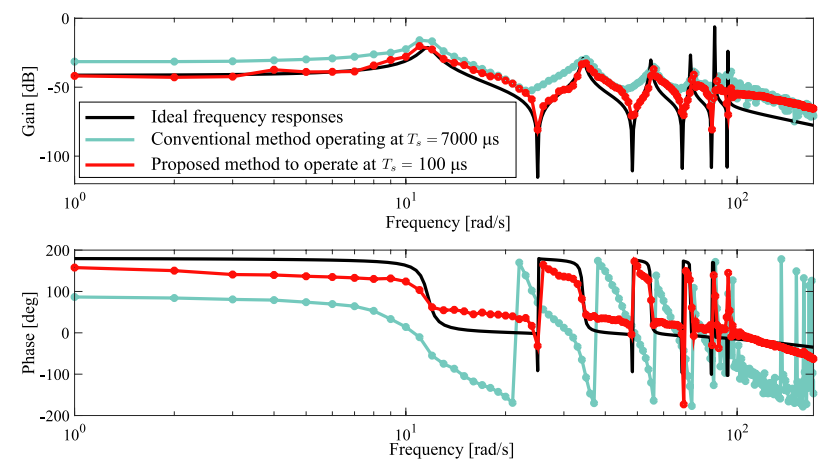

Fig. 11. Frequency responses haptic reproduction

period. Only the proposed method realizes broadband admittance control. The broadband admittance control accurately reproduces the gain and phase characteristics, Thus, the reproducibility is improved by the FDTD method.

\section{Conclusions}

In a conventional system, the matrix exponential function is implemented by the Pade approximation, which has large errors and large discretization time for higher-order systems.

In particular, the Pade approximation exponentially increases discretization time when the system matrix, matrix $A$, becomes large. The proposed method does not degrade the accuracy of higher order systems. Furthermore, becouse the discretization time is small, high speed discretization is possible. In the experiments, the FDTD method is applied to the haptic simulator, and the time-variant system is accurately reproduced in real-time. The effectiveness of the proposed method is demonstrated by simulations and experiments.

\section{References}

( 1 ) M. Kumar and T. Shen: "Cycle-to-cycle transient model of 4-stroke combustion engines", 4th International Conference On Simulation And Modeling Methodologies, Technologies And Applications (SIMULTECH), pp.745750 (2014)

( 2 ) S. Inaishi, H. Eguchi, and T. Yamashita: "Robust Stabilization Based on Eigenvalue Assignment", Transactions of the Society of Instrument and Control Engineers, Vol.33, No.4, pp.241-246 (1997)

( 3 ) N. Kobayashi, K. Nonami, and I. Yasuzumi: "Attitude Control of Free-Flying Space Robot with Discrete Time Sliding Mode Control Algorithm", Transactions of the Society of Instrument and Control Engineers, Vol.32, No.8, pp.1206-1211 (1996)

( 4 ) M. Otsuki, S. Shibata, and K. Yoshida: "Design of Robust Positioning Control Input Considering Structured Uncertainty for Time-Varying Flexible Structure", Transactions of the Japan Society of Mechanical Engineers Series C, Vol.72, No.721, pp.2861-2868 (2006)

( 5 ) S. Hara, K. Nakamura, and T. Nnrikiyo: "Positioning and Vibration Control Using Nonstationary Optimal Regulator for Time-Varying Vibration Systems", Transactions of the Japan Society of Mechanical Engineers Series C, Vol.68, No.665, pp.44-51 (2002)

( 6 ) M. Otsuki, K. Yoshida, K. Nngata, H. Kimura, and T. Nnkagawa: "Vibration Control for Rope-Sway of Elevator of High-Rise Building: Application of 
Nonstationary Optimal Control to Time-Varying Flexible System", Transactions of the Japan Society of Mechanical Engineers Series C, Vol.68, No.676, pp.3589-3596 (2002)

( 7 ) K. Fujita and S. Hokamoto: "Feedback Control of Orbital Parameters for a Rigid Satellite under Nonholonomic Constraint", Journal of The Japan Society for Aeronautical and Space Sciences, Vol.56, No.648, pp.1-7 (2008)

( 8 ) H. Nishimura, H. Tanemura, and K. Nonami: "Gain-Scheduled Positioning Control for Varying Rope Length in Travelling Cranes", Transactions of the Japan Society of Mechanical Engineers Series C, Vol.62, No.599, pp.26922698 (1996)

( 9 ) M. Ogata, I. Nishino, and K. Kajihara: "An Advent of Simulators", The Journal of The Institute of Image Information and Television Engineers, Vol.56, No.12, pp.1912-1917 (2002)

(10) M. Nerio and Y. Chiku: "Introduction to Simulation Technology for Analysis of Human Factor", Journal of The Society of Instrument and Control Engineers, Vol.45, No.8, pp.726-730 (2006)

(11) D. Pilastro, R. Oboe, and T. Shimono: "A Nonlinear Adaptive Compliance Controller for Rehabilitation", IEEJ J. Industry Applications, Vol.5, No.2, pp.123-131 (2016)

(12) K. Watanabe: "Approximated Calculation Method of the Transition Matrix Using Pade's Approximate Function of $\exp (\mathrm{x})$ :", Transactions of the Society of Instrument and Control Engineers, Vol.10, No.1, pp.36-44 (1974)

(13) J. Shimazaki: "On Error Evaluation of Pade Approximants to exp(z)", Transactions of the Society of Instrument and Control Engineers, Vol.19, No.2, pp.179-181 (1983)

(14) K. Yee: "Numerical solution of initial boundary value problems involving maxwell's equations in isotropic media", IEEE Antennas and Propagation Society, Vol.14, No.3, pp.302-307 (1996)

(15) A. Taflove and S.C. Hagness: "Computational electrodynamics: The FiniteDifference Time-Domain Method 3-rd ed.", Artech House on Demand (2005)

(16) T. Tsuchiya: "Sound Field Simulation and Sound Field Rendering", IEICE ESS Fundamentals Review, Vol.10, No.3, pp.206-218 (2017)

(17) Y. Asai, Y. Yokokura, and K. Ohishi: "Mechanical Admittance Realization Control Based on Feedforward Compensation for Fine Realization of Impedance", IEEJ J. Industry Applications, Vol.6, No.2, pp.83-90 (2017)

(18) T. Tsuru and S. Haruyama: "A Guide to the Impedance Measurement for Corrosion Research", Corrosion Engineering, Vol.35, No.5, pp.296-301 (1986)

\section{Appendix}

In this section, the discretization accuracy of the FDTD method is theoretically evaluated in the continuous time domain. The discrete time state differential equations are inversely converted to continuous time.

\section{Discretization Error of FDTD Method}

For instance, thecontinuous target system is defined as follows:

$$
A_{c}=\left[\begin{array}{cc}
0 & k_{1} \\
-\frac{1}{m_{1}} & 0
\end{array}\right]
$$

Eq. (A1) is discretized by the FDTD method as follows:

$$
A_{d}^{F D T D}=\left[\begin{array}{cc}
1-\frac{k_{l} T_{s}^{2}}{m_{l}} & k_{l} T_{s} \\
-\frac{T_{s}}{m_{l}} & 1
\end{array}\right]
$$

where the eigenvalues of $\lambda_{1}$ and $\lambda_{2}$ are as follows:

$$
\begin{aligned}
& \lambda_{1}=\frac{-k_{1} T_{s}^{2}+2 m_{1}+\sqrt{T_{s}{ }^{4} k_{1}^{2}-4 k_{1} T_{s}^{2} m_{1}}}{2 m_{1}} \cdots \\
& \lambda_{2}=\frac{-k_{1} T_{s}^{2}+2 m_{1}-\sqrt{T_{s}{ }^{4} k_{1}^{2}-4 k_{1} T_{s}^{2} m_{1}}}{2 m_{1}} . \cdots
\end{aligned}
$$

The eigenvalues are converted from discrete time to continuous time as follows:

$$
\begin{aligned}
& \eta_{1}=\ln \left(\lambda_{1}\right) / T_{s} \\
& \eta_{2}=\ln \left(\lambda_{2}\right) / T_{s} .
\end{aligned}
$$

The eigenvalues of the FDTD method, which are inversely transformed by the ideal eigenvector $T$, are converted into a continuous matrix. The matrix of the FDTD method $A_{c}^{F D T D}$ converted to continuous time is as follows:

$$
\begin{aligned}
& A_{c}^{F D T D}=T\left[\begin{array}{cc}
\eta_{1} & 0 \\
0 & \eta_{2}
\end{array}\right] T^{-1}=\left[\begin{array}{ll}
A_{11} & A_{12} \\
A_{21} & A_{22}
\end{array}\right] \\
& =\left[\begin{array}{c}
\frac{1}{2 T_{s}}\left(-2 \ln (2)+\ln \left(2 \lambda_{1}\right)+\ln \left(2 \lambda_{2}\right)\right) \\
-\frac{\sqrt{-m_{1} k_{1}}}{2 k_{1} T_{s} m_{1}}\left(-\ln \left(2 \lambda_{1}\right)+\ln \left(2 \lambda_{2}\right)\right)
\end{array}\right. \\
& \left.\begin{array}{c}
-\frac{m_{1} k_{1}}{2 \sqrt{-m_{1} k_{1}} T_{s}}\left(-\ln \left(2 \lambda_{1}\right)+\ln \left(2 \lambda_{2}\right)\right) \\
\frac{1}{2 T_{s}}\left(-2 \ln (2)+\ln \left(2 \lambda_{1}\right)+\ln \left(2 \lambda_{2}\right)\right)
\end{array}\right] .
\end{aligned}
$$

To compare the difference between Eq. (A7) and Eq. (A1), Eq. (A7) is expanded by the Taylor expansion with $T_{s}$, as follows:

$$
\begin{aligned}
& A_{11}=0 \ldots \ldots \ldots \ldots \ldots \ldots \ldots \ldots \ldots \ldots \ldots \ldots \ldots \ldots \\
& A_{12}=k_{1} \underbrace{+\frac{k_{1}^{2} T_{s}^{2}}{24 m_{1}}+\frac{3 k_{1}{ }^{3} T_{s}{ }^{4}}{640 m_{1}^{2}}+\frac{5 k_{1}{ }^{4} T_{s}{ }^{6}}{7168 m_{1}^{3}}}_{\text {Error terms }} \cdots \\
& A_{21}=-\frac{1}{m_{1}} \underbrace{-\frac{k_{1} T_{s}{ }^{2}}{24 m_{1}^{2}}-\frac{3 k_{1}^{2} T_{s}^{4}}{640 m_{1}^{3}}-\frac{5 k_{1}^{3} T_{s}^{6}}{7168 m_{1}^{4}}}_{\text {Error terms }} \\
& A_{22}=0 .
\end{aligned}
$$

Here, the first term of each element represents Eq. (A1). In addition, $T_{s}$ is approximated to zero, and Eq. (A7) represents the original system of Eq. (A1) as follows:

$$
\lim _{T_{s} \rightarrow 0} A_{c}^{F D T D}=\left[\begin{array}{cc}
0 & k_{1} \\
-\frac{1}{m_{1}} & 0
\end{array}\right]=A_{c}
$$

\section{Discretization Error of the Pade Approximation}

As with the FDTD method, the discretization accuracy of the first-order Pade approximation is evaluated in the continuous time domain. The matrix $A_{c}$ discretized by the first-order Pade approximation is as follows:

$$
A_{d}^{\text {Pade }}=\left[\begin{array}{cc}
\frac{-k_{1} T_{s}{ }^{2}+4 m_{1}}{k_{1} T_{s}{ }^{2}+4 m_{1}} & \frac{4 k_{1} T_{s} m_{1}}{k_{1} T_{s}{ }^{2}+4 m_{1}} \\
-\frac{4 T_{s}}{k_{1} T_{s}{ }^{2}+4 m_{1}} & \frac{-k_{1} T_{s}^{2}+4 m_{1}}{k_{1} T_{s}{ }^{2}+4 m_{1}}
\end{array}\right]
$$

where the eigenvalues of $\lambda_{1}^{\prime}$ and $\lambda_{2}^{\prime}$ are as follows:

$$
\begin{aligned}
& \lambda_{1}^{\prime}=\frac{-k_{1} T_{s}^{2}+4 \sqrt{-m_{1} k_{1}} T_{s}+4 m_{1}}{k_{1} T_{s}^{2}+4 m_{1}} \\
& \lambda_{2}^{\prime}=\frac{-k_{1} T_{s}^{2}-4 \sqrt{-m_{1} k_{1}} T_{s}-4 m_{1}}{k_{1} T_{s}^{2}+4 m_{1}} .
\end{aligned}
$$

Eq. (A14) and Eq. (A14) are converted to continuous time by Eq. (A5). The eigenvalues of the first-order Pade approximation, which are inversely transformed by the ideal eigenvector $T$, are converted into a continuous matrix. The matrix of the first-order Pade approximation $A_{c}^{\text {Pade }}$ converted to continuous time is as follows:

$$
A_{c}^{\text {Pade }}=T\left[\begin{array}{cc}
\eta_{1}^{\prime} & 0 \\
0 & \eta_{2}^{\prime}
\end{array}\right] T^{-1}=\left[\begin{array}{cc}
A_{11}^{\prime} & A_{12}^{\prime} \\
A_{21}^{\prime} & A_{22}^{\prime}
\end{array}\right]
$$




$$
\begin{gathered}
{\left[\begin{array}{c}
\frac{1}{2 T_{s}} \ln \left(\lambda_{1}^{\prime}\right)+\frac{1}{2 T_{s}} \ln \left(\lambda_{2}^{\prime}\right) \\
\frac{\sqrt{-m_{1} k_{1}}}{2 k_{1} T_{s} m_{1}} \ln \left(\lambda_{1}^{\prime}\right)-\frac{\sqrt{-m_{1} k_{1}}}{2 k_{1} T_{s} m_{1}} \ln \left(\lambda_{2}^{\prime}\right) \\
\frac{m 1 k 1}{2 \sqrt{-m_{1} k_{1} T_{s}}} \ln \left(\lambda_{1}^{\prime}\right)-\frac{m_{1} k_{1}}{2 \sqrt{-m_{1} k_{1}} T_{s}} \ln \left(\lambda_{2}^{\prime}\right) \\
\frac{1}{2 T s} \ln \left(\lambda_{1}^{\prime}\right)+\frac{1}{2 T_{s}} \ln \left(\lambda_{2}^{\prime}\right) \\
\ldots \ldots \ldots \ldots \ldots \ldots .
\end{array} . .\right.}
\end{gathered}
$$

Similarly, the Taylor expansion of $A_{c}^{\text {Pade }}$, which is inversely transformed, becomes as follows:

$$
\begin{aligned}
& A_{11}^{\prime}=0 \\
& A_{12}^{\prime}=k_{1} \underbrace{-\frac{k_{1}{ }^{2} T_{s}{ }^{2}}{12 m_{1}}+\frac{k_{1}{ }^{3} T_{s}{ }^{4}}{80 m_{1}{ }^{2}}-\frac{k_{1}{ }^{4} T_{s}{ }^{6}}{448 m_{1}{ }^{3}}}_{\text {Error terms }} \\
& A_{21}^{\prime}=-\frac{1}{m_{1}} \underbrace{+\frac{k_{1} T_{s}{ }^{2}}{12 m_{1}{ }^{2}}-\frac{k_{1}{ }^{2} T_{s}{ }^{4}}{80 m_{1}{ }^{3}}+\frac{k_{1}{ }^{3} T_{s}{ }^{6}}{448 m_{1}{ }^{4}}} \cdots \\
& A_{22}^{\prime}=0
\end{aligned}
$$

Here, the first term of each element represents Eq. (A1). In addition, $T_{s}$ is approximated to zero. Eq. (A16) represents the original system of Eq. (A1) as follows:

$$
\lim _{T_{s} \rightarrow 0} A_{c}^{\text {Pade }}=\left[\begin{array}{cc}
0 & k_{1} \\
-\frac{1}{m_{1}} & 0
\end{array}\right]=A_{c}
$$

According to Eq. (A1), Eq. (A12) and Eq. (A21), both the FDTD method and the Pade approximation are correct.

\section{Comparison}

This paper compares the coefficients of Taylor expansion. A comparison of $A_{12}$ and $A_{12}^{\prime}$ is shown as follows:

(Left side : (Right side :

1st Pade approximation) FDTD method)

$$
\begin{aligned}
& k_{1}=k_{1} \ldots \ldots \ldots \ldots \ldots . \\
& \left|-\frac{k_{1}{ }^{2} T_{s}{ }^{2}}{12 m_{1}}\right|>\left|+\frac{k_{1}{ }^{2} T_{s}{ }^{2}}{24 m_{1}}\right| \ldots . \\
& \left|+\frac{k_{1}{ }^{3} T_{s}{ }^{4}}{80 m_{1}{ }^{2}}\right|>\left|+\frac{3 k_{1}{ }^{3} T_{s}{ }^{4}}{640 m_{1}{ }^{2}}\right| \ldots \\
& \left|-\frac{k_{1}{ }^{4} T_{s}{ }^{6}}{448 m_{1}{ }^{3}}\right|>\left|+\frac{5 k_{1}{ }^{4} T_{s}{ }^{6}}{7168 m_{1}{ }^{3}}\right| \ldots
\end{aligned}
$$

A comparison of $A_{21}$ and $A_{21}^{\prime}$ is shown as follows:

(Left side : (Right side :

1st Pade approximation) FDTD method)

$$
\begin{aligned}
& -\frac{1}{m_{1}}=-\frac{1}{m_{1}} \ldots \ldots \ldots \ldots \ldots \ldots \ldots \ldots \ldots \ldots \\
& \left|+\frac{k_{1} T_{s}^{2}}{12 m_{1}^{2}}\right|>\left|-\frac{k_{1} T_{s}^{2}}{24 m_{1}^{2}}\right|
\end{aligned}
$$

$$
\begin{aligned}
& \left|-\frac{k_{1}^{2} T_{s}^{4}}{80 m_{1}^{3}}\right|>\left|-\frac{3 k_{1}^{2} T_{s}^{4}}{640 m_{1}^{3}}\right| \ldots \\
& \left|+\frac{k_{1}^{3} T_{s}^{6}}{448 m_{1}{ }^{6}}\right|>\left|-\frac{5 k_{1}^{3} T_{s}^{6}}{7168 m_{1}{ }^{4}}\right| .
\end{aligned}
$$

The Pade approximationis represented on the left side and the FDTD method is represented on the right side. In $A_{12}, A_{12}^{\prime}$ and $A_{21}, A_{21}^{\prime}$ terms, the FDTD method has all its coefficients, which are smaller than those of the first-order Pade approximation. In other words, the discretization accuracy of the FDTD method is higher than that of the Pade approximation.

Hirotaka Muto (Student Member) received B.E. degrees in electrical

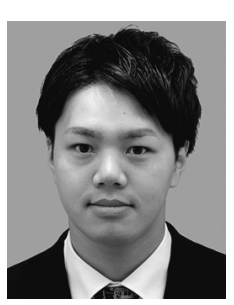
engineering from Nagaoka University of Technology, Nagaoka, Japan, in 2017. Now he is a candidate of the M.E. degree in Electrical, Electronics and Information Engineering. His research interests include motion control and real-world haptics. He is a member of the Institute of Electrical Engineering of Japan (IEEJ), and the Robotics Society of Japan (RSJ).

Yuki Yokokura

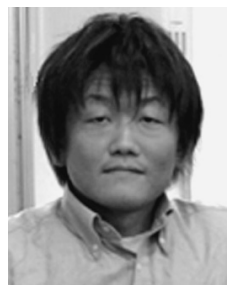

(Member) received the B.E. and M.E. degrees in electrical engineering from Nagaoka University of Technology, Niigata, Japan, in 2007 and 2009, respectively. In 2011, he received Ph.D. degree in integrated design engineering from Keio University, Yokohama, Japan. From 2010 to 2011, he was a Japan Society for the Promotion of Science (JSPS) Research Fellow (DC2 and PD). He was a Visiting Fellow at Keio University, and a Postdoctoral Fellow at Nagaoka University of Technology in 2011. Since 2012, he has been an Assistant Professor with Nagaoka University of Technology. His research interests include motion control, motor drive, and real-world haptics.

Kiyoshi Ohishi (Fellow) received the B.E., M.E., and Ph.D. degrees

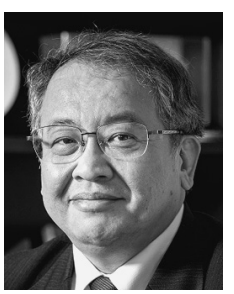
in electrical engineering from Keio University, Japan, in 1981, 1983, and 1986, respectively. Since 1993, he has been with Nagaoka University of Technology, Nagaoka, Japan. Now, he becomes a full Professor from 2003 and becomes a vice President from 2016. His research interests include motion control, mechatronics, robotics and power electronics. He is a Fellow Member of IEEE from 2015, whose contributions are the Development of Fast and Robust Motion Control based on Force Sensing Technology. From 2004, he has been an AdCom Member at large of IEEE IES Society for 12 years. From 2016, now, he is a senior AdCom Member of IEEE IES Society. He obtained the outstanding paper award of IECON1985. Moreover, he received Best Paper Awards at IECON2002 and IECON2004, and he received "Best paper award" of EPE-PEMC2008. He organized IEEE/IES IECON2015 and was a General Chair of IECON2015. Moreover, he organized IEEE IES AMC2018 and AMC2010. He was the General Chairs of AMC2018 and AMC2010. He was a Technical Program Chair of IEEE/IES AMC2006. 